\title{
Sleep quality and OPRM1 polymorphisms: a cross-sectional study among opioid-naive individuals
}

\author{
Zalina Zahari1,2,*, Muslih Abdulkarim Ibrahim ${ }^{2,3}$, Nurfadhlina Musa², Soo Choon Tan², \\ Nasir Mohamad ${ }^{2,4}$, Rusli Ismail ${ }^{2,4}$
}

\begin{abstract}
${ }^{1}$ Faculty of Pharmacy, Universiti Sultan Zainal Abidin (UniSZA), Besut Campus, Terengganu, Malaysia, ${ }^{2}$ Pharmacogenetics and Novel Therapeutics Cluster, Institute for Research in Molecular Medicine (INFORMM), Universiti Sains Malaysia (USM), Kelantan, Malaysia, ${ }^{3}$ Department of Pharmacology and Toxicology, College of Pharmacy, Hawler Medical University, Hawler, Iraq, ${ }^{4}$ Faculty of Medicine, Universiti Sultan Zainal Abidin (UniSZA), Medical Campus, Terengganu, Malaysia
\end{abstract}

\begin{abstract}
Opioidergic system involves in regulation of sleep and wakefulness. It is possible, therefore, that genetic polymorphisms in OPRM1 influence sleep quality. This study investigated the association of OPRM1 polymorphisms with subjective sleep quality among opioid-naive individuals. This cross-sectional observational study involved 161 opioid-naive males (mean age $=27.74$ years; range: $18-63$ years). Subjective sleep quality was assessed with the translated and validated Malay version of the Pittsburgh Sleep Quality Index (PSQI). DNA was extracted from whole blood and subjected to polymerase chain reaction (PCR)-genotyping for two OPRM1 polymorphisms (118A $>\mathrm{G}$ and IVS2+691G $>\mathrm{C})$. Subjects with combined $118 \mathrm{~A}$ and $I V S 2+691 G$ alleles (AC haplotype) had significantly lower PSQI scores [mean $(\mathrm{SD})=4.29(1.76)]$ compared to those without the haplotype $[4.99(2.50)](\mathrm{p}=0.004)$. On the other hand, subjects with combined heterozygous genotype (GC/AG diplotype) had significantly higher PSQI scores compared to those without the diplotype [6.04 (2.48) vs 4.54 (2.22), $\mathrm{p}=0.004]$. In opioid-naive individuals, AC haplotype and GC/AG diplotype for the $118 \mathrm{~A}>\mathrm{G}$ and IVS2 $+691 \mathrm{G}>\mathrm{C}$ polymorphisms of OPRM1 are associated with better and poorer sleep quality, respectively.
\end{abstract}

Keywords: Sleep-Wake Transition Disorders. Sleep quality. Sleep/genetic. Polymorphism. Pittsburgh Sleep Quality Index. OPRM1. Opioid receptors $/ \mu$ analysis.

\section{INTRODUCTION}

Poor sleep quality has been reported in general population. In German, $36 \%$ of the general population slept badly (Hinz et al., 2017). A survey conducted in China reported that the overall prevalence of insomnia was $26.6 \%$ among 26,851 subjects from the general population (Tang et al., 2017). A study among 794 medical students in Malaysia found that $16.1 \%$ reported bad sleep quality (Zailinawati et al., 2009). In another study, a higher prevalence of poor sleep quality (32.9\%) was reported among 1,118 Malaysian tertiary students (Lai, Say, 2013). Shift work, sleep disorders, socioeconomic status, aging, anxiety and the number of chronic disease, amongst others,

\footnotetext{
*Correspondence: Z. Zahari. Faculty of Pharmacy, Universiti Sultan Zainal Abidin (UniSZA), Besut Campus, 22200 Besut, Terengganu, Malaysia. Tel: + 609 6993119; Fax: + 609 6993004. E-mail: zalinazahari@unisza.edu.my or zalina240678@yahoo.com
}

were found to be significantly associated with poor sleep quality (Guo et al., 2013; Patel et al., 2010; Luo et al., 2013; Hinz et al., 2017; Tang et al., 2017). Poor sleep quality is often associated with accidents (Garbarino et al., 2001; Powell et al., 2007), and an increased in total healthcare and lost productivity costs (Sarsour et al., 2011). In addition, sleep problems are associated with a decreased in health related quality of life (Schubert et al., 2002; Rashid et al., 2012) and have been reported as a risk factor for all-cause mortality (Marshall et al., 2008). More recently, a community-based population study has shown that moderate-to-severe sleep apnea is independently associated with a large increased risk of all-cause mortality, incident stroke, and cancer incidence and mortality (Marshall et al., 2014).

Genetic and environmental factors have been implicated in sleep and sleep problems (Heath et al., 1990; Lessov-Schlaggar et al., 2008; Watson et al., 2006; Genderson et al., 2013). Research suggests that genetic 
differences accounted for at least $33 \%$ of the variance in sleep quality and sleep disturbance and $40 \%$ of the variance in sleep pattern (Heath et al., 1990). Recently, a twin research has highlighted the importance of genes on the Pittsburgh Sleep Quality Index (PSQI) and the seven components of the PSQI (Genderson et al., 2013). Genetic association studies have provided some progress in understanding the genetic control of sleep and heritability of sleep disorders (Varvarigou et al., 2011; Evans et al., 2013; Palagini, Biber, Riemann, 2014; Wisor, Kilduff, 2005; Millstein et al., 2011; Andretic, Franken, Tafti, 2008). Individual differences in regulation of sleep and wakefulness can be explained by variation within specific genes, and single nucleotide polymorphisms (SNPs) or combinations of SNP alleles that tend to be inherited together (haplotypes) may be associated with sleep in healthy populations and among sleep-disordered patients (Andretic, Franken, Tafti, 2008).

The endogenous opioid peptide $\beta$-endorphin is known to be involved in the regulation of sleep and wakefulness (King et al., 1981). Pharmacological studies have shown that the naturally-occurring opioid peptide, $\beta$-endorphin, interacts preferentially with opioid receptor, mu 1 (OPRM1). A previous study has shown that $\beta$-endorphin and morphine injected intraventricularly produced insomnia in cats (King et al., 1981). Their findings showed that deep slow wave sleep (SWS) was sharply inhibited, rapid eye movement (REM) sleep was entirely suppressed, and light slow wave sleep, occurring in brief, isolated episodes, became the most abundant stage of sleep. They also found that an opioid receptor antagonist, naloxone, injected subcutaneously reversed the effects of $\beta$-endorphin and morphine on the two stages of SWS, but it did not counteract the REM-suppressant effect of either $\beta$-endorphin or morphine (King et al., 1981). This data may indicate an involvement of an inner opioid in the regulation of sleep-wake cycle (King et al., 1981), and further research suggest the existence of somnogenic effect of opioidergic system in the caudal nucleus tractus solitarius (NTS) (Reinoso-Barbero, Andrés, 1995).

Later, electroacupuncture (EA) of Anmian acupoints has been shown to increase non-rapid eye movement (NREM) sleep, but not REM sleep, during the dark period in rats (Cheng et al., 2011). This data suggest that stimulation of the opiodergic neurons to increase the concentrations of $\beta$-endorphin and the involvement of the $\mu$-opioid receptors may also be a mechanism by which acupuncture affects sleep (Cheng et al., 2011). It is possible, therefore, some of the OPRM1 polymorphisms that affect the density and function, and consequently the signaling efficacy of $\mu$-opioid receptors may contribute to inter-individual variations in the sleep quality. The most frequently studied polymorphism of $O P R M 1,118 \mathrm{~A}>\mathrm{G}$ (dbSNP rs 1799971, Asn40Asp) polymorphism, is found in exon 1. It may affect the $\mu$-opioid receptor $\mathrm{N}$-glycosylation and reduced stability of the receptor (Huang et al., 2012). IVS2+691G $>$ C (dbSNP rs2075572) polymorphism is found within intron 2 at $691 \mathrm{bp}$ downstream of exon 2 (Xin, Wang, 2002; Hoehe et al., 2000; Lötsch, Geisslinger, 2006). It may change the regulation of the expression of OPRM1 gene and may also cause formation of different isoforms of human $\mu$-opioid receptor (Hoehe et al., 2000; Wendel, Hoehe, 1998). Unfortunately, the association between OPRM1 polymorphisms and sleep quality is unknown and requires exploration. Examining the influence of genetic polymorphisms on sleep quality will be informative in terms of gaining a deeper understanding of sleep and sleep problems. In this study, we aimed to investigate the influence of OPRM1 polymorphisms on sleep quality among opioid-naive individuals.

\section{MATERIAL AND METHODS}

\section{Participants}

One hundred and sixty one healthy volunteers who had taken part in a pharmacogenetics study conducted by the Institute for Research in Molecular Medicine (INFORMM), USM, Kota Bharu, Kelantan, and who satisfied inclusion and exclusion criteria were recruited from the local community between March and October 2013. Opioid-naive individual was defined as individual who have not taken any opioids including morphine and methadone to the best of their knowledge and have had two consecutive negative urine screenings results for illicit drugs. Inclusion criteria were: 1) Malay male aged more than 18 years; 2) Free of acute medical, surgical and psychiatric illness; and 3) Free of regular use of alcohol. The exclusion criteria were: 1) individuals who were currently taking illicit benzodiazepines, cannabinoids and barbiturates; 2) individuals on regular anticonvulsants, neuroleptics or analgesics; 3 ) individuals with chronic or ongoing acute pain; 4) individuals with a history of analgesics ingestion within three days before the study; and 5) individuals with severe cognitive impairment which may interfere with sleep assessments and/or communication.

Written informed consent was obtained from each subject after a complete description of the study. The study was registered with the National Institutes of Health (NIH) of the Ministry of Health (MOH), Malaysia (available at www.nmrr.gov.my, National Medical Research Register 
(NMRR) number: NMRR-13-524-16614). The study was evaluated and approved by the Human Research Ethics Committee (HREC), Universiti Sains Malaysia (USM) in Kelantan, Malaysia (Reference number: USMKK/PPP/ $\operatorname{JEPeM}(253.3$ [14]).

\section{Assessment of subjective sleep quality using the Malay version of the Pittsburgh Sleep Quality Index (PSQI-M)}

Subjective sleep quality was measured by the Malay version of the Pittsburgh Sleep Quality Index (PSQI-M) (Buysse et al., 1989). The PSQI is a validated questionnaire to measure subjective sleep quality and disturbances during the previous month that has been translated into several languages including Malay. The Malay translation of this questionnaire was performed by MAPI Research Trust and permission for use the Malay version of the Pittsburgh Sleep Quality Index - PSQI was obtained from the author at the University of Pittsburgh (Buysse et al., 1989). The PSQI contains 19 items that are included in scoring. The 19 individual items are used to generate seven component scores: subjective sleep quality, sleep latency, sleep duration, habitual sleep efficiency, sleep disturbances, use of sleep medications, and daytime dysfunction. Each of the seven component scores is determined based on scoring guidelines, with the seven component scores each with a potential range of $0-3$, where ' 3 ' reflects the negative extreme on the Likert Scale. The sum of these seven component scores yields one global score of subjective sleep quality with a potential range of $0-21$, with higher scores represent poorer subjective sleep quality (i.e. a global PSQI score of $>5$ is associated with poor sleep quality) (Buysse $e t$ al., 1989).

\section{Genotyping methods for detection of OPRM1 polymorphisms}

Subjects provided $5 \mathrm{~mL}$ venous blood sample, and genomic DNA was extracted from $200 \mu$ of whole blood by use of the QIAamp ${ }^{\circledR}$ DNA Blood Mini Kit (Qiagen Gmbh, Hilden, Germany). Allele-specific multiplex polymerase chain reaction (PCR) was used to detect OPRM1 polymorphisms [118A $>\mathrm{G}$ (dbSNP rs 1799971) and IVS2+691G $>$ C (dbSNP rs2075572)] (Mohamed Nazar, 2013; Zahari et al., 2015; Zahari et al., 2016b, Zahari et al., 2016a). All reactions were performed on the Applied Biosystems ${ }^{\circledast}$ Veriti ${ }^{\circledast}$ 96-Well Thermal Cycler (Applied Biosystems, USA). A complete PCR method is available upon request.

\section{Statistical analysis}

Sample size was calculated prior the start of the study based on the Cohen sample size table (Cohen, 1992), using medium population effect size (ES) of 0.50 assuming a two-tailed $5 \%$ type I error rate and $80 \%$ power. The required sample size per group was 64 alleles or subjects for comparisons of means of two groups (under allelic additive model, genotype dominant and recessive model). $O P R M 1$ haplotypes and diplotypes were constructed based on the expectation-maximization (EM) algorithm using the population genetic data analytical program, Golden Helix SNP and Variation Suite 7 (SVS 7, version 7.3.1; Golden Helix Inc., Bozeman, MT, USA). The sum of seven component scores of the PSQI was calculated as the global score for subjective sleep quality. Means and standard deviations were calculated for the global score of the PSQI. Independent t-test and one-way ANOVA test were used to compare the mean global PSQI scores between OPRM1 polymorphisms ( $118 \mathrm{~A}>\mathrm{G}$ and IVS $2+691 \mathrm{G}>\mathrm{C})$ according to their genotypes and allelic additive models, genotype dominant and recessive models, haplotypes and diplotypes where appropriate. Haplotypes and diplotypes with frequencies less than $10 \%$ were pooled, since rare or minor haplotypes and diplotypes provide little additional information while increasing the degrees of freedom. Data analyses were done after all genotyping of the subjects was completed. There were no missing data for all the subjects included in this study. Given the exploratory nature of this study and only one gene was tested (Belfer et al., 2013), correction for multiple testing was not performed for the exploratory statistical analyses. Statistical analysis was carried out using SPSS/Win software (Version 22, SPSS, Inc., Chicago, IL, USA). A $p$ value $<0.05$ was considered significant.

\section{RESULTS}

\section{Descriptive statistics}

A total of 161 opioid-naive individuals fulfilled inclusion and exclusion criteria, gave informed consent and completed the study. The mean age of study participants was 27.74 years [standard deviation $(\mathrm{SD})=10.32$; range: 18-63 years]. The mean body mass index (BMI) was $24.82 \mathrm{~kg} / \mathrm{m}^{2}\left(\mathrm{SD}=5.33\right.$; range: $\left.15-45 \mathrm{~kg} / \mathrm{m}^{2}\right)$. The mean PSQI score was $4.76(\mathrm{SD}=2.31$, range $0-13)$, slightly below a cut-off score of 5, thus indicating good overall sleep quality (Buysse et al., 1989). Specifically, 68.9\% $(N=111)$ had PSQI scores of $\leq 5$, indicating they were 'good sleepers'. 


\section{OPRM1 polymorphisms}

The $118 A / G$ and $I V S 2+691 G / C$ alleles of OPRM1 were successfully amplified from the DNA of 161 opioid-naive subjects. The allele frequencies for $118 \mathrm{G}$ and $I V S 2+691 C$ were $53.1 \%$ and $85.1 \%$, respectively. The combination of the individual polymorphisms into OPRM1 haplotype pairs revealed the presence of 7 diplotypes. The most common haplotype pair was AC/GC $(N=54,33.5 \%)$, followed by $\mathrm{GC} / \mathrm{GC}(N=45,28.0 \%)$, $\mathrm{GC} / \mathrm{AG}(N=23,14.3 \%)$, and $\mathrm{AC} / \mathrm{AG}(N=21,13.0 \%)$.

\section{Association of OPRM1 polymorphisms with PSQI scores}

Table I shows that subjects with homozygous 118
AA genotype had lower PSQI scores compared to those without 118 AA genotype (118 AG/GG genotype) (4.30 vs 4.90), but the difference was not statistically significant $(\mathrm{p}=0.167)$.

Subjects with the heterozygous IVS2+691 GC genotype had the highest PSQI scores among the three IVS2+691G $>$ C genotypes (Table I). Subjects with the IVS2+691G allele (IVS2+691 GG/GC genotype) had higher PSQI scores compared to those without the allele (IVS2+691 CC genotype) (5.26 vs 4.55), but the difference again did not reach statistical significance $(\mathrm{p}=0.079)$.

In view of this, we performed haplotype and diplotype analysis constructed from the two OPRM1 polymorphisms. Haplotype analysis revealed a significant difference of the mean PSQI scores between subjects with AC haplotype and those without this haplotype

TABLE I - Association between 118A $>\mathrm{G}$ and IVS2+691G $>$ C polymorphisms and PSQI scores in opioid-naive Malay Males

\begin{tabular}{|c|c|c|c|c|c|c|}
\hline Polymorphism & $N$ & $(\%)$ & Mean & SD & Test statistic (df) & $p$ value $^{\mathrm{c}}$ \\
\hline \multicolumn{7}{|l|}{$118 \mathrm{~A}>\mathrm{G}$} \\
\hline \multicolumn{7}{|c|}{ Genotype $(N=161)$} \\
\hline AA & 37 & 23.0 & 4.30 & 1.61 & $1.11(2,158)^{\mathrm{a}}$ & 0.331 \\
\hline $\mathrm{AG}$ & 77 & 47.8 & 4.81 & 2.22 & & \\
\hline GG & 47 & 29.2 & 5.04 & 2.84 & & \\
\hline \multicolumn{7}{|c|}{ Allele $(N=322)$} \\
\hline A & 151 & 46.9 & 4.56 & 1.95 & $-1.50(313)^{\mathrm{b}}$ & 0.134 \\
\hline G & 171 & 53.1 & 4.94 & 2.57 & & \\
\hline \multicolumn{7}{|c|}{ Dominant model } \\
\hline $\mathrm{AA}$ & 37 & 23.0 & 4.30 & 1.61 & $-1.39(159)^{\mathrm{b}}$ & 0.167 \\
\hline $\mathrm{AG}+\mathrm{GG}$ & 124 & 77.0 & 4.90 & 2.47 & & \\
\hline \multicolumn{7}{|c|}{ Recessive model } \\
\hline $\mathrm{AA}+\mathrm{AG}$ & 114 & 70.8 & 4.64 & 2.05 & $-0.88(67)^{b}$ & 0.382 \\
\hline GG & 47 & 29.2 & 5.04 & 2.84 & & \\
\hline \multicolumn{7}{|l|}{$\overline{I V S} 2+691 \mathrm{G}>\mathrm{C}$} \\
\hline \multicolumn{7}{|c|}{ Genotype $(N=161)$} \\
\hline GG & 1 & 0.6 & 4.00 & - & $1.71(2,158)^{\mathrm{a}}$ & 0.184 \\
\hline GC & 46 & 28.6 & 5.28 & 2.34 & & \\
\hline $\mathrm{CC}$ & 114 & 70.8 & 4.55 & 2.28 & & \\
\hline \multicolumn{7}{|c|}{ Allele $(N=322)$} \\
\hline G & 48 & 14.9 & 5.23 & 2.31 & $1.54(320)^{\mathrm{b}}$ & 0.124 \\
\hline $\mathrm{C}$ & 274 & 85.1 & 4.68 & 2.30 & & \\
\hline \multicolumn{7}{|c|}{ Dominant model } \\
\hline GG & 1 & 0.6 & 4.00 & - & $-0.33(159)^{b}$ & 0.743 \\
\hline $\mathrm{GC}+\mathrm{CC}$ & 160 & 99.4 & 4.76 & 2.31 & & \\
\hline \multicolumn{7}{|c|}{ Recessive model } \\
\hline $\mathrm{GG}+\mathrm{GC}$ & 47 & 29.2 & 5.26 & 2.33 & $1.77(159)^{\mathrm{b}}$ & 0.079 \\
\hline $\mathrm{CC}$ & 114 & 70.8 & 4.55 & 2.28 & & \\
\hline
\end{tabular}


Sleep quality and OPRM1 polymorphisms: a cross-sectional study among opioid-naive individuals

TABLE I - Association between 118A > G and IVS2+691G $>$ C polymorphisms and PSQI scores in opioid-naive Malay Males (cont.)

\begin{tabular}{|c|c|c|c|c|c|c|}
\hline Polymorphism & $N$ & $(\%)$ & Mean & SD & Test statistic (df) & $p$ value $^{\mathrm{c}}$ \\
\hline \multicolumn{7}{|l|}{ Haplotype $(N=322)^{\mathrm{d}}$} \\
\hline $\mathrm{GC}$ & 169 & 52.5 & 4.92 & 2.55 & $2.66(3,318)^{\mathrm{a}}$ & 0.048 \\
\hline $\mathrm{AC}$ & 105 & 32.6 & 4.29 & 1.76 & & \\
\hline AG & 46 & 14.3 & 5.17 & 2.22 & & \\
\hline GG & 2 & 0.6 & 6.50 & 4.95 & & \\
\hline $\mathrm{GC}$ & 169 & 52.5 & 4.92 & 2.55 & $3.68(2,319)^{\mathrm{a}}$ & 0.026 \\
\hline $\mathrm{AC}$ & 105 & 32.6 & 4.29 & 1.76 & & \\
\hline Combined $\mathrm{AG}$ and $\mathrm{GG}$ & 48 & 14.9 & 5.23 & 2.31 & & \\
\hline $\mathrm{GC}$ & 169 & 52.5 & 4.92 & 2.55 & $1.32(313)^{b}$ & 0.187 \\
\hline Not GC & 153 & 47.5 & 4.58 & 1.99 & & \\
\hline $\mathrm{AC}$ & 105 & 32.6 & 4.29 & 1.76 & $-2.90(278)^{\mathrm{b}}$ & 0.004 \\
\hline Not AC & 217 & 67.4 & 4.99 & 2.50 & & \\
\hline $\mathrm{AG}$ & 46 & 14.3 & 5.17 & 2.22 & $1.33(320)^{\mathrm{b}}$ & 0.186 \\
\hline Not AG & 276 & 85.7 & 4.69 & 2.31 & & \\
\hline \multicolumn{7}{|l|}{ Diplotype $(N=161)$} \\
\hline $\mathrm{AC} / \mathrm{GC}$ & 54 & 33.5 & 4.28 & 1.89 & $2.83(4,156)^{\mathrm{a}}$ & 0.027 \\
\hline $\mathrm{GC} / \mathrm{GC}$ & 45 & 28.0 & 4.98 & 2.79 & & \\
\hline $\mathrm{GC} / \mathrm{AG}$ & 23 & 14.3 & 6.04 & 2.48 & & \\
\hline $\mathrm{AC} / \mathrm{AG}$ & 21 & 13.0 & 4.33 & 1.62 & & \\
\hline Others $^{\mathrm{e}}$ & 18 & 11.2 & 4.50 & 2.09 & & \\
\hline $\mathrm{AC} / \mathrm{GC}$ & 54 & 33.5 & 4.28 & 1.89 & $-1.89(159)^{\mathrm{b}}$ & 0.061 \\
\hline Not AC/GC & 107 & 66.5 & 5.00 & 2.46 & & \\
\hline $\mathrm{GC} / \mathrm{GC}$ & 45 & 28.0 & 4.98 & 2.79 & $0.66(64)^{b}$ & 0.509 \\
\hline Not GC/GC & 116 & 72.0 & 4.67 & 2.10 & & \\
\hline $\mathrm{GC} / \mathrm{AG}$ & 23 & 14.3 & 6.04 & 2.48 & $2.96(159)^{\mathrm{b}}$ & 0.004 \\
\hline Not GC/AG & 138 & 85.7 & 4.54 & 2.22 & & \\
\hline $\mathrm{AC} / \mathrm{AG}$ & 21 & 13.0 & 4.33 & 1.62 & $-0.90(159)^{\mathrm{b}}$ & 0.368 \\
\hline Not AC/AG & 140 & 87.0 & 4.82 & 2.39 & & \\
\hline
\end{tabular}

$N$, number of subject/allele/haplotype/diplotype; SD, standard deviation. ${ }^{a} F$-statistic using one-way ANOVA test; ${ }^{b} t$-statistic using independent t-test; ${ }^{\mathrm{c}} p$ value is significant at $<0.05 ;{ }^{\mathrm{d}}$ Haplotype patterns were constructed from the two OPRM1 polymorphisms $(118 \mathrm{~A}>\mathrm{G}$ and IVS2 $+691 \mathrm{G}>\mathrm{C})$; ${ }^{\mathrm{e}}$ Diplotype with frequency less than $10.0 \%$ was pooled under 'others' (included AG/GG and GC/GG)

$[t(278)=-2.90, p=0.004]$. Subjects with the AC haplotype had significantly lower PSQI scores compared to those without the haplotype (4.29 vs 4.99).

There was also a significant difference of the mean PSQI scores between subjects with the combined heterozygous genotype (GC/AG diplotype) and those without this diplotype $(\mathrm{p}=0.004)$. Subjects with the GC/AG diplotype had significantly higher PSQI scores compared to those without the diplotype (6.04 vs 4.54).

\section{DISCUSSION}

The present study aimed to investigate the associations between $O P R M 1$ polymorphisms and sleep quality in opioid-naive population. This study revealed that the mean PSQI scores was significantly lower in carriers of the AC haplotype than those without the haplotype. On the other hand, this study found that the mean PSQI scores was significantly higher in carriers of the GC/AG diplotype than those without the diplotype. From these data, it is suggested that variations in OPRM1 may influence sleep quality even in the opioid-naive population. Previous research using the classical twin design has highlighted the contribution of genes to subjective sleep quality (Genderson et al., 2013). Sleep quality was measured using the PSQI in 1218 middle-aged twin men from the 
Vietnam Era Twin Study of Aging (VETSA) (mean age $=55.4$ years; range: $51-60$ ). They found that $34 \%$ of variability in the global PSQI score was due to additive genetic effects (heritability). Similarly, the heritability of poor sleep (i.e. a dichotomous measure based on the cut-off of global PSQI $>5$ ) was $31 \%$. Previous molecular genetic studies have begun to identify genetic variations related to sleep-wake behavior includes $3111 \mathrm{~T} / \mathrm{C} \mathrm{SNP}$ (rs1801260) of the Circadian Locomotor Output Cycles Kaput (CLOCK) gene (Benedetti et al., 2007) and variable-number tandem-repeat (VNTR) polymorphism of the period circadian clock 3 gene (PER3) (Viola et al., 2007). Also, there are a number of recent genome-wide association analyses (GWAS) studies on sleep quality and quantity (Lane et al., 2017; Jones et al., 2016; Byrne et al., 2013).

Among the identified polymorphisms within the OPRM1 gene, $118 \mathrm{~A}>\mathrm{G}$ polymorphism is the most frequently studied in the literatures. An in vitro study has demonstrated that the $118 G$ allele altered the $\beta$-endorphin binding affinity (Bond et al., 1998). The $118 G$ allele showed a 3 -fold increase in $\beta$-endorphin binding at the receptor than the most common allelic form of the receptor (Bond et al., 1998). $\beta$-endorphin involves in the regulation of sleep and wakefulness, and administration of $\beta$-endorphin has been shown to produce insomnia (King et al., 1981). It is possible, therefore, the $118 G$ allele carriers have higher $\beta$-endorphin binding at the OPRM1 and consequently, resulted in poorer sleep quality compared to those without the allele. In our study, although results did not reach statistical significance, $118 G$ allele carriers (118 AG/GG genotype) had higher PSQI scores when compared with the $118 G$ allele non-carriers (118 AA genotype).

A more significant influence of OPRMI polymorphisms on sleep quality was observed under haplotype and diplotype analysis. We found that subjects with AC haplotype (i.e. carriers of combined $118 \mathrm{~A}$ and $I V S 2+691 G$ alleles) had $14 \%$ significantly lower PSQI scores compared to non-carriers of this haplotype. We also found that subjects with $\mathrm{GC} / \mathrm{AG}$ diplotype (i.e. carriers of combined 118 AG and IVS2+691 GC genotypes) had 33\% significantly higher PSQI scores compared to non-carriers of this diplotype. Unfortunately, the exact molecular mechanism regarding the effects of AC haplotype and $\mathrm{GC} /$ AG diplotype on the sleep quality cannot be determined from the current study. To the best of our knowledge, data on the influence of the OPRMI haplotype and diplotype on sleep quality among opioid-naive individuals is not available for reference. However, previous studies have indicated that the function of the OPRM1 is under the influence OPRM1 polymorphisms (Xin, Wang, 2002;
Hoehe et al., 2000; Lötsch, Geisslinger, 2006). Based on our results, we suggest that the AC haplotype and $\mathrm{GC} /$ AG diplotype may affect OPRM1 expression or function (or both), and resulted in altered binding affinity between endogenous opioid peptides and the OPRM1, and hence haplotype and diplotype differences may contribute to inter-individual differences in sleep-wakefulness effects of endogenous opioid peptide $\beta$-endorphin.

It has been previously demonstrated that the $\mathrm{AC} /$ AG diplotype for the $118 \mathrm{~A}>\mathrm{G}$ and IVS $2+691 \mathrm{G}>\mathrm{C}$ polymorphisms of OPRM1 is associated with better sleep quality among opioid-dependent patients on MMT (Zahari et al., 2016a). However, in opioid-naive individuals, no significant difference of the mean PSQI scores was observed between subjects with the AC/AG diplotype and those without this diplotype ( $p=0.368)$. It is highly possible that the OPRM1 affects sleep by acting on different mechanisms in opioid-naive individuals and opioid-dependent patients (Wang et al., 2012; GarcíaGarcía, Drucker-Colín, 1999). At this juncture, it is difficult to explain the differential effects on sleep quality among opioid-naive individuals and opioid-dependent patients with these polymorphisms and further studies are needed.

The findings of the present study have therapeutic implications. The main findings here were that the contribution of OPRM1 polymorphisms influences to the sleep quality among opioid-naive individuals varied between genotype, haplotype and diplotype analysis. Most notably that subjects with GC/AG diplotype $(N=23$, $14.3 \%$ ) had the PSQI scores of $>5$, indicating they were 'poor sleepers', and their PSQI scores was significantly higher than those without the diplotype. These results provide an initial prediction on sleep quality for individuals with GC/AG diplotype which enables prescription of sleep medications for individuals with the diplotype. Furthermore, the clinician may use genotyping results of OPRMI in making decision about pharmacotherapeutic strategies in terms of the choice of medications to be prescribed, and dose and/or route of administration of drugs for treatment of postoperative sleep disturbance among patients undergoing surgery. Indeed, this study demonstrated that individuals with $\mathrm{GC} / \mathrm{AG}$ diplotype are more susceptible to poor sleep compared to individuals without the diplotype. It is possible that the good sleep is more difficult to be achieved in individuals with GC/AG diplotype. Thus, the results of the current study provide new information regarding the genetic factors that may be consider when establishing clinical recommendations or a suitable treatment protocol for providing sleep disorder treatment and management. However, separate analysis of individual components of the 
PSQI showed that only sleep disturbance was significantly associated with $\mathrm{GC} / \mathrm{AG}$ diplotype $[t(\mathrm{df})=2.14(26.8), p=$ 0.042]. Individuals with $\mathrm{GC} / \mathrm{AG}$ diplotype had $25 \%$ higher sleep disturbance score compared to individuals without the diplotype (1.35 vs 1.08). Early detection and intervention of sleep disturbances can help reduce the morbidity and mortality associated with this and helps increase patient's quality of life.

Certain limitations of this study must be recognized. This study did not use an objective sleep evaluation test such as polysomnography (PSG) and did not obtain data on serum concentrations of $\beta$-endorphin and supporting data on the functional effects of $O P R M 1$ polymorphisms $(118 \mathrm{~A}>\mathrm{G}$ and IVS2+691G $>\mathrm{C})$, haplotypes or diplotypes on OPRM1 expression or function (or both). Only males were included but we aimed to reduce the confounding effects of gender on sleep quality (Fatima et al., 2016). In view that correction for multiple comparisons was not performed for exploratory statistical analyses, caution is required when reviewing these analyses. The sample size was another limitation, but we considered our study as more explorative in nature and replication by other investigators and further studies with larger sample sizes are encouraged. This study lacked a multivariate approach. Non-genetics sleep-related factors were not reported, and this study was design to exclude subjects with chronic medical and psychiatric illness such as chronic pain, depression and anxiety that are associated with sleep disorder because our focus was to look into pharmacogenetics factors associated with sleep quality among opioid-naive individuals. However, we did not find any significant differences between individuals with GC/ AG diplotype and those without the diplotype in terms of age and BMI, therefore they were well matched with respect to age and BMI.

Further studies are needed to study other OPRM1 polymorphisms and genetic variations of other sleep related-genes, and to obtain data on endogenous $\beta$-endorphin concentration and data on the functional effects of AC haplotype and GC/AG diplotype on OPRM1 expression and/or function.

In summary, our study indicates that the AC haplotype and $\mathrm{GC} / \mathrm{AG}$ diplotype for the $118 \mathrm{~A}>\mathrm{G}$ and IVS $2+691 \mathrm{G}>\mathrm{C}$ polymorphisms of OPRMI are associated with better and poorer sleep quality, respectively among opioid-naive individuals. Our study may be considered explorative in nature and it would require replication by other investigators. Nevertheless, these results provide a starting point for a better understanding of genetic contributions to sleep quality which may help to improve diagnosis and treatment of sleep disturbance.

\section{ACKNOWLEDGEMENTS}

We wish to thank Prof. Howard McNulty of the Institute of Pharmacy and Bio-medical Sciences University of Strathclyde Glasgow UK for English language editing and proof reading of this article. We are grateful to Nur Amalina Che Rahim and Wan Izzati Mariah Binti Wan Hassan from Department of Pharmacy, Hospital Universiti Sains Malaysia, Kubang Kerian, Kelantan, Malaysia; and all the members of Pharmacogenetics and Novel Therapeutics Cluster, Institute for Research in Molecular Medicine (INFORMM), Universiti Sains Malaysia (USM) for their support and valuable suggestions during the study. The study was funded by the Universiti Sains Malaysia (USM) grant under the 'Research University Cluster (RUC)' Grant No.1001.PSK.8620014, under the project; Application of Personalised Methadone Therapy Methadone Maintenance Therapy (PMT for MMT).

\section{REFERENCES}

Andretic R, Franken P, Tafti M. Genetics of sleep. Ann Rev Genet. 2008;42:361-88.

Belfer I, Segall SK, Lariviere WR, Smith SB, Dai F, Slade GG, et al. Pain modality- and sex-specific effects of COMT genetic functional variants. Pain. 2013;154(8):1368-76.

Benedetti F, Dallaspezia S, Fulgosi MC, Lorenzi C, Serretti A, Barbini B, et al. Actimetric evidence that CLOCK 3111 T/C SNP influences sleep and activity patterns in patients affected by bipolar depression. Am J Med Genet B Neuropsychiatr Genet. 2007;144B(5):631-5.

Bond C, Laforge KS, Tian M, Melia D, Zhang S, Borg L, et al. Single-nucleotide polymorphism in the human mu opioid receptor gene alters beta-endorphin binding and activity: possible implications for opiate addiction. Proc Natl Acad Sci U S A. 1998;95(16):9608-13.

Buysse DJ, Reynolds CF 3rd, Monk TH, Berman SR, Kupfer DJ. The Pittsburgh Sleep Quality Index: a new instrument for psychiatric practice and research. Psychiatry Res. 1989;28(2):193-213.

Byrne EM, Gehrman PR, Medland SE, Nyholt DR, Heath AC, Madden PaF, et al. A genome-wide association study of sleep habits and insomnia. Am J Med Genet B Neuropsychiatr Genet. 2013;162B(5):439-51. 
Cheng CH, Yi PL, Lin JG, Chang FC. Endogenous opiates in the nucleus tractus solitarius mediate electroacupuncture-induced sleep activities in rats. Evid Based Complement Alternat Med. 2011;2011(art.159209):1-11.

Cohen J. A power primer. Psychol Bull. 1992;112(1):155-9.

Evans DS, Parimi N, Nievergelt CM, Blackwell T, Redline S, Ancoli-Israel S, et al. Common genetic variants in ARNTL and NPAS2 and at chromosome 12 p13 are associated with objectively measured sleep traits in the elderly. Sleep. 2013;36(3):431-46.

Fatima Y, Doi SaR, Najman JM, Mamun AA. Exploring gender difference in sleep quality of young adults: findings from a large population study. Clinical Medicine \& Research. 2016;14(3/4):138-44.

Garbarino S, Nobili L, Beelke M, De Carli F, Ferrillo F. The contributing role of sleepiness in highway vehicle accidents. Sleep. 2001;24(2):203-6.

García-García F, Drucker-Colín R. Endogenous and exogenous factors on sleep-wake cycle regulation. Prog Neurobiol. 1999;58(4):297-314.

Genderson MR, Rana BK, Panizzon MS, Grant MD, Toomey $\mathrm{R}$, Jacobson $\mathrm{KC}$, et al. Genetic and environmental influences on sleep quality in middle-aged men: a twin study. J Sleep Res. 2013;22(5):519-26.

Guo Y, Liu Y, Huang X, Rong Y, He M, Wang Y, et al. The effects of shift work on sleeping quality, hypertension and diabetes in retired workers. PLoS One. 2013;8(8):e71107.

Heath AC, Kendler KS, Eaves LJ, Martin NG. Evidence for genetic influences on sleep disturbance and sleep pattern in twins. Sleep. 1990;13(4):318-35.

Hinz A, Glaesmer H, Brähler E, Löffler M, Engel C, Enzenbach $\mathrm{C}$, et al. Sleep quality in the general population: psychometric properties of the Pittsburgh Sleep Quality Index, derived from a German community sample of 9284 people. Sleep Med. 2017;30:57-63.

Hoehe MR, Kopke K, Wendel B, Rohde K, Flachmeier C, Kidd KK, et al. Sequence variability and candidate gene analysis in complex disease: association of mu opioid receptor gene variation with substance dependence. Hum Mol Genet. 2000;9(19):2895-908.
Huang P, Chen C, Mague SD, Blendy JA, Liu-Chen LY. A common single nucleotide polymorphism A118G of the mu opioid receptor alters its $\mathrm{N}$-glycosylation and protein stability. Biochem J. 2012;441(1):379-86.

Jones SE, Tyrrell J, Wood AR, Beaumont RN, Ruth KS, Tuke MA, et al. Genome-wide association analyses in 128,266 individuals identifies new morningness and sleep duration loci. PLoS Genet. 2016;12(8):e1006125.

King C, Masserano JM, Codd E, Byrne WL. Effects of betaendorphin and morphine on the sleep-wakefulness behavior of cats. Sleep. 1981;4(3):259-62.

Lai PP, Say YH. Associated factors of sleep quality and behavior among students of two tertiary institutions in Northern Malaysia. Med J Malaysia. 2013;68(3):195-203.

Lane JM, Liang J, Vlasac I, Anderson SG, Bechtold DA, Bowden J, et al. Genome-wide association analyses of sleep disturbance traits identify new loci and highlight shared genetics with neuropsychiatric and metabolic traits. Nat Genet. 2017;49(2):274-81.

Lessov-Schlaggar CN, Bliwise DL, Krasnow RE, Swan GE, Reed T. Genetic association of daytime sleepiness and depressive symptoms in elderly men. Sleep. 2008;31(8):1111-7.

Lötsch J, Geisslinger G. Relevance of frequent mu-opioid receptor polymorphisms for opioid activity in healthy volunteers. Pharmacogenomics J. 2006;6(3):200-10.

Luo J, Zhu G, Zhao Q, Guo Q, Meng H, Hong Z, et al. Prevalence and risk factors of poor sleep quality among Chinese elderly in an urban community: results from the Shanghai Aging Study. PLoS One. 2013;8(11):e81261.

Marshall NS, Wong KK, Cullen SR, Knuiman MW, Grunstein RR. Sleep apnea and 20-year follow-up for all-cause mortality, stroke, and cancer incidence and mortality in the Busselton Health Study cohort. J Clin Sleep Med. 2014;10(4):355-62.

Marshall NS, Wong KK, Liu PY, Cullen SR, Knuiman MW, Grunstein RR. Sleep apnea as an independent risk factor for all-cause mortality: the Busselton Health Study. Sleep. 2008;31(8):1079-85.

Millstein J, Winrow CJ, Kasarskis A, Owens JR, Zhou L, Summa KC, et al. Identification of causal genes, networks, and transcriptional regulators of REM sleep and wake. Sleep. 2011;34(11):1469-77. 
Mohamed Nazar NI. Therapeutic drug monitoring in methadone maintenance therapy (MMT): an evaluation of genetic factors influencing clinical outcomes and serum concentrations of methadone. [Ph.D. Thesis]. Pusat Pengajian Sains Perubatan: Universiti Sains Malaysia, 2013.

Palagini L, Biber K, Riemann D. The genetics of insomnia-evidence for epigenetic mechanisms? Sleep Med Rev. 2014;18(3):225-35.

Patel NP, Grandner MA, Xie D, Branas CC, Gooneratne N. "Sleep disparity" in the population: poor sleep quality is strongly associated with poverty and ethnicity. BMC Public Health. 2010;10:art.475.

Powell NB, Schechtman KB, Riley RW, Guilleminault C, Chiang RP, Weaver EM. Sleepy driver near-misses may predict accident risks. Sleep. 2007;30(3):331-42.

Rashid A, Ong EK, Wong ESY. Sleep quality among residents of an old folk's home in Malaysia. Iran J Nurs Midwifery Res. 2012;17(7):512-9.

Reinoso-Barbero F, Andrés I. Effects of opioid microinjections in the nucleus of the solitary tract on the sleep-wakefulness cycle states in cats. Anesthesiology. 1995;82(1):144-52.

Sarsour K, Kalsekar A, Swindle R, Foley K, Walsh JK. The association between insomnia severity and healthcare and productivity costs in a health plan sample. Sleep. 2011;34(4):443-50.

Schubert CR, Cruickshanks KJ, Dalton DS, Klein BE, Klein R, Nondahl DM. Prevalence of sleep problems and quality of life in an older population. Sleep. 2002;25(8):889-93.

Tang J, Liao Y, Kelly BC, Xie L, Xiang Y-T, Qi C, et al. Gender and regional differences in sleep quality and insomnia: a general population-based study in Hunan Province of China. Sci Rep. 2017; 7 :art.43690

Varvarigou V, Dahabreh IJ, Malhotra A, Kales SN. A review of genetic association studies of obstructive sleep apnea: field synopsis and meta-analysis. Sleep. 2011;34(11):1461-8.

Viola AU, Archer SN, James LM, Groeger JA, Lo JC, Skene DJ, et al. PER3 polymorphism predicts sleep structure and waking performance. Curr Biol. 2007;17(7):613-8.
Wang SC, Tsou HH, Chen CH, Chen YT, Ho IK, Hsiao CF, et al. Genetic polymorphisms in the opioid receptor mul gene are associated with changes in libido and insomnia in methadone maintenance patients. Eur Neuropsychopharmacol. 2012;22(10):695-703.

Watson NF, Goldberg J, Arguelles L, Buchwald D. Genetic and environmental influences on insomnia, daytime sleepiness, and obesity in twins. Sleep. 2006;29(5):645-9.

Wendel B, Hoehe MR. The human mu opioid receptor gene: 5 ' regulatory and intronic sequences. J Mol Med (Berl). 1998;76(7):525-32.

Wisor JP, Kilduff TS. Molecular genetic advances in sleep research and their relevance to sleep medicine. Sleep. 2005;28(3):357-67.

Xin L, Wang ZJ. Bioinformatic analysis of the human mu opioid receptor (OPRM1) splice and polymorphic variants. AAPS PharmSci. 2002;4(4, art.E23):39-45.

Zahari Z, Lee CS, Ibrahim MA, Musa N, Mohd Yasin MA, Lee $\mathrm{YY}$, et al. The AC/AG diplotype for the $118 \mathrm{~A}>\mathrm{G}$ and IVS2 + $691 \mathrm{G}>\mathrm{C}$ polymorphisms of OPRM1 gene is associated with sleep quality among opioid-dependent patients on methadone maintenance therapy. Pain Ther. 2016a;5(1):43-54.

Zahari Z, Lee CS, Ibrahim MA, Musa N, Yasin MA, Lee YY, et al. The opposing roles of IVS2+691 CC genotype and AC/ AG diplotype of $118 \mathrm{~A}>\mathrm{G}$ and IVS $2+691 \mathrm{G}>\mathrm{C}$ of OPRM 1 polymorphisms in cold pain tolerance among opioid-dependent Malay males on methadone therapy. Pain Ther. 2015;4(2):17996.

Zahari Z, Lee CS, Lee YY, Ibrahim MA, Musa N, Mohd Yasin $\mathrm{MA}$, et al. $118 \mathrm{~A}>\mathrm{G}$ and IVS2 $+691 \mathrm{G}>\mathrm{C}$ polymorphisms of OPRM1 gene have no influence on cold-pain sensitivity among healthy opioid-naive Malay males. Int J Pharm Pharm Sci. 2016b;8(7):73-80.

Zailinawati AH, Teng CL, Chung YC, Teow TL, Lee PN, Jagmohni KS. Daytime sleepiness and sleep quality among Malaysian medical students. Med J Malaysia. 2009;64(2):10810 .

Received for publication on $04^{\text {th }}$ May 2017 Accepted for publication on $09^{\text {th }}$ June 2017 